\title{
Impact of intraprosthetic drilling on the strength of the femoral stem in periprosthetic fractures: A finite element investigation
}

Proc IMechE Part H:

$\mathrm{J}$ Engineering in Medicine

2016, Vol. 230(7) 675-681

(c) IMechE 2016

Reprints and permissions:

sagepub.co.uk/journalsPermissions.nav DOI: $10.1177 / 09544$ I1916647078

pih.sagepub.com

(S)AGE

\author{
Stephan Brand', Michael Bauer², Maximilian Petri', Julian Schrader², \\ Hans J Maier ${ }^{2}$, Christian Krettek' and Thomas Hassel ${ }^{2}$
}

\begin{abstract}
Treatment of periprosthetic femur fractures after total hip arthroplasty remains a major challenge in orthopedic surgery. Recently, a novel surgical technique using intraprosthetic screw fixation has been suggested. The purpose of this study was to evaluate the influence of drilling the femoral hip stem on integrity and strength of the implant. The hypothesis was that intraprosthetic drilling and screw fixation would not cause the load limit of the prosthesis to be exceeded and that deformation would remain within the elastic limit. A sawbone model with a conventional straight hip stem was used and a Vancouver $C$ periprosthetic fracture was created. The fracture was fixed with a nine-hole less invasive stabilization system plate with two screws drilled and inserted through the femoral hip stem. Three different finite element models were created using ANSYS software. The models increased in complexity including joint forces and stress risers from three different dimensions. A variation of drilling positions was analyzed. Due to the complexity of the physiological conditions in the human femur, the most complex finite element model provided the most realistic results. Overall, significant changes in the stresses to the prosthesis caused by the drilling procedure were observed. While the stresses at the site of the bore hole decreased, the load increased in the surrounding stem material. This effect is more pronounced and further the holes were apart, and it was found that increasing the number of holes could counteract this. The maximum load was still found to be in the area of the prosthesis neck. No stresses above the load limit of titanium alloy were detected. All deformations of the prosthesis stem remained in the elastic range. These results may indicate a potential role for intraprosthetic screw fixation in the future treatment of periprosthetic femur fractures.
\end{abstract}

\section{Keywords}

Finite element analysis, intraprosthetic fixation, ANSYS

Date received: 26 March 2015; accepted: 5 April 2016

\section{Introduction}

With a continuously increasing number of primary hip arthroplasties, the incidence of periprosthetic fractures is also increasing. ${ }^{1-3}$ The risk of sustaining a periprosthetic fracture estimates to be about $0.3 \%-2 \%$ in the first year after implantation. ${ }^{3-7}$

Depending on the fracture type and classification, a vast majority of these fractures require surgical treatment. Although different fixation methods are available, the best surgical approach in fixation is still controversial and depends on the type of fracture, quality of bone stock, patient's age, and comorbidities. ${ }^{8}$

For surgical decision-making, the Vancouver classification is most commonly used. It considers the type of fracture, the stability of the stem, and the quality of the surrounding bone. Fractures with loose stems (Vancouver B2) should be treated by revision arthro-

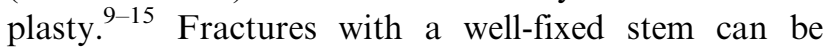
treated by prosthesis-retaining osteosynthesis. Osteosynthesis treatment options for periprosthetic fractures include plate fixation, ${ }^{16-21}$ cerclage wiring, ${ }^{22-24}$

\footnotetext{
'Trauma Department, Hannover Medical School (MHH), Hannover, Germany

${ }^{2}$ Institute of Materials Science, Leibniz University of Hannover, Hannover, Germany
}

\section{Corresponding author:}

Stephan Brand, Trauma Department, Hannover Medical School (MHH), Carl-Neuberg-Street I, D-30625 Hannover, Germany.

Email: brand.stephan@mh-hannover.de 
external fixation, ${ }^{25,26}$ and combinations of the techniques. Osteoporotic bone and lack of bony anchoring are major concerns for any of these techniques. ${ }^{27,28}$

Most recently, a novel surgical technique using the stable prosthesis as the strongest part of the proximal femur for intraprosthetic screw fixation has been suggested. $^{27,28}$ This method was shown to significantly increase primary stability compared to conventional techniques. Concerns about high drilling temperatures potentially causing osteonecrosis could be sorted out. $^{29,30}$

However, the impact of drilling the hip stem on implant integrity has not been investigated yet. Drill holes may weaken the strength of the prosthesis, potentially leading to material failure and breaking of the prosthesis.

The purpose of this study was to evaluate the stem integrity depending on different drilling procedures using the finite element (FE) method. FE analysis allows for evaluation of both the drill hole positioning and the number of drill holes in the stem and their impact on implant strength.

The hypothesis was that intraprosthetic drilling and screw fixation would not cause the load limit of the prosthesis to be exceeded and that deformation would remain within the elastic limit.

\section{Materials and methods}

Synthetic replicas of the human left femur (Sawbones, Vashon Island, WA, USA) were used for this study. The structure and biomechanical properties of these replicas are comparable to a human femur with cortical and cancellous bone. In all simulations, the same femur was used for consistency.

A conventional cementless straight hip stem made of a titanium wrought alloy (TiAl6V4) (ASTM F136) (Ecofit, Implantcast, Buxtehude, Germany) of size $10 \mathrm{~mm}$ was implanted into the sawbone. The head of the prosthesis (diameter $28 \mathrm{~mm}$ ) also consisted of TiAl6V4 with a thin titanium nitride (TiN) coating.

A standardized Vancouver $\mathrm{C}$ periprosthetic fracture was then created. For osteosynthesis, a less invasive stabilization system (LISS) implant was used (Synthes GmbH, Oberdorf, Switzerland). The LISS-DF plate 5.0 is made of TiAl6Nb7 (ASTM F 1295). The LISS includes nine holes with an overall length of $236 \mathrm{~mm}$.

Two standardized holes with a diameter of $5.0 \mathrm{~mm}$ and a depth of $10 \mathrm{~mm}$ each were drilled perpendicularly into the prosthetic femoral stem using a standard 4.3-mm drill. Two regular 5.0-mm locking screws were inserted. Two variations of drilling were tested. In variation one, the distance between the centers of the holes was $20 \mathrm{~mm}$ according to the drill hole distance of the LISS plate; in variation two, the distance between the centers was $40 \mathrm{~mm}$ (Figure 1). The first drill was set up $14 \mathrm{~mm}$ below the angulation of the straight stem (Figure 1).

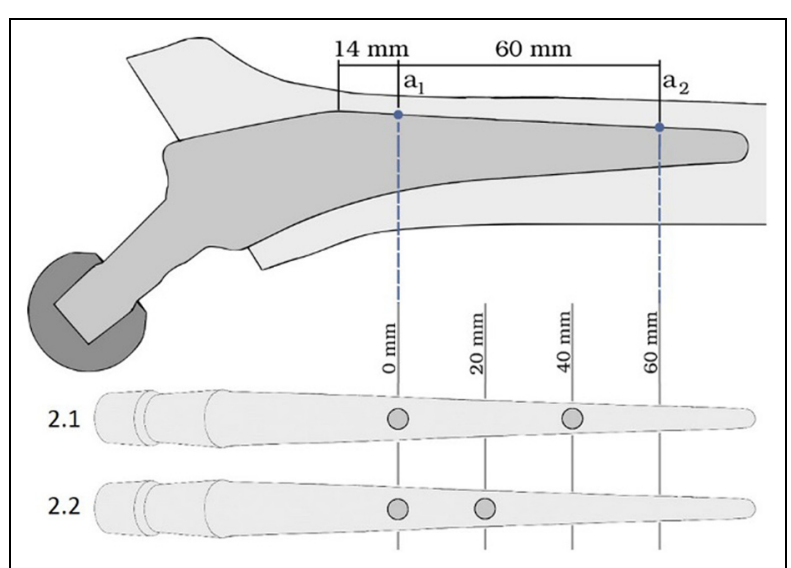

Figure I. Two variations of the drilling setup into the prosthesis.

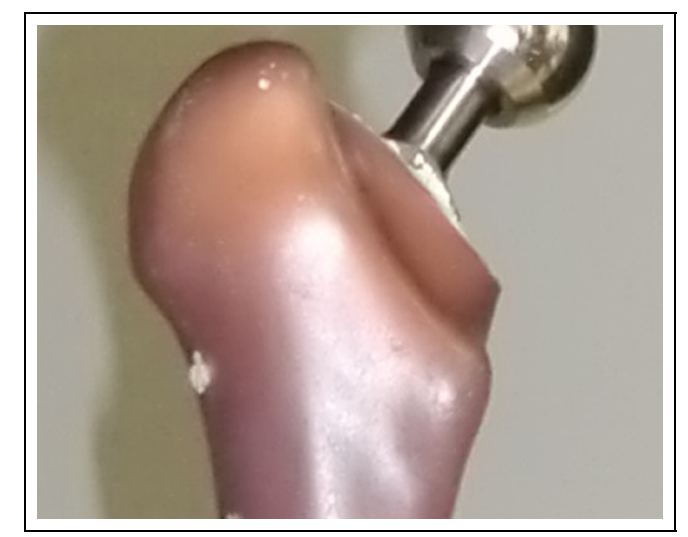

Figure 2. Head distant restriction IIB. ${ }^{31,32}$

Each of the components was scanned using a portable REVscan 3d-scanner (Fa. Creaform, Lévis, Québec, Canada) with XV elements Version 1.1 (Fa. Creaform). The scanning resolution was $0.5 \mathrm{~mm}$. After scanning, the component geometries were imported into SolidWorks 2014 (Fa. Dassault Systèmes, Velizy Villacoublay, France) and the items were combined to form an assembly. The assembly was then transferred with an integrated import tool to the FE program ANSYS Workbench 14.0 (ANSYS Inc., Canonsburg, PA, USA).

After scanning the topography, the geometries were revised by the Design-Modeler of ANSYS. Several small areas of the scanned parts prevented a uniform mesh. Thus, these areas were manually edited and joined together to form large, continuous areas. Then, the boundary conditions were defined and the material parameters were inserted into ANSYS. Finally, the contact points were defined, and simulation was performed.

It is noteworthy that the pure titanium coating is not considered in the FE model. The resection plane for removal of the femoral head corresponds to the head distant restriction IIB in Figure 2. 


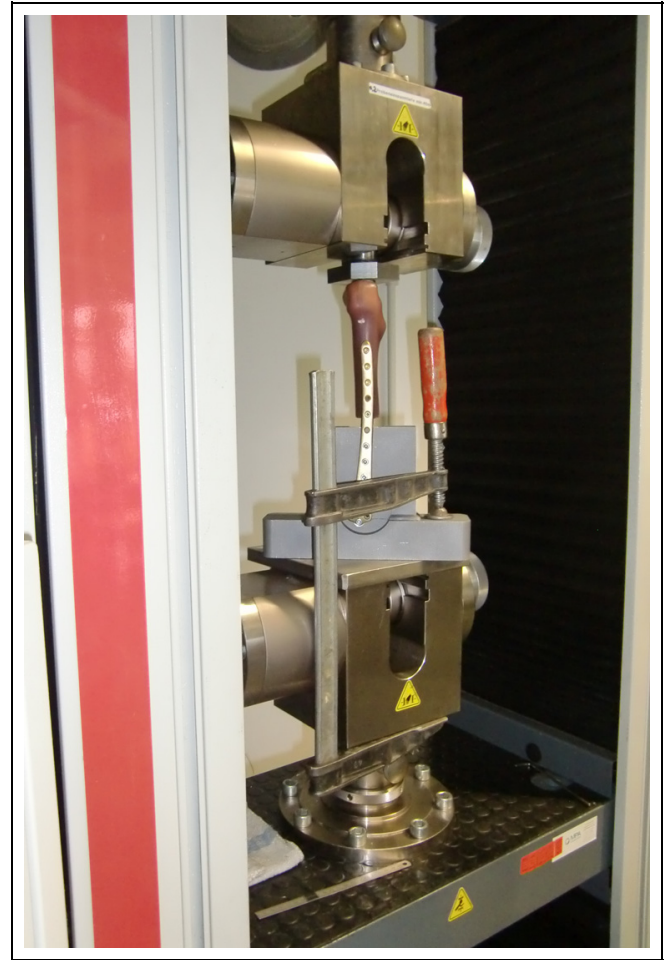

Figure 3. Test design. ${ }^{27,28}$

The cementless prosthesis was regularly fixed into the femur by maintaining the caput-collum-diaphyseal (CCD) angle.

The surface of the femur was then scanned, and the considered length in this simulation was $200 \mathrm{~mm}$ using the material data of a human cortical bone.

The osteosynthesis plate was not conventionally screwed onto the femur, but rather anchored into the stem of the prosthesis as previously described ${ }^{27,28}$ (Figure 3). In our simulation, a simplification of the screw was inserted; screws and threaded bushings are replaced by connecting pins with a length of $20 \mathrm{~mm}$ and a diameter of $5.5 \mathrm{~mm}$. The boreholes of the osteosynthesis plate were adjusted accordingly and the contact defined as a composite FE function.

For creating the mesh of the model components, the element types SOLID186 and SOLID187 were used. These element types describe tetrahedral elements with quadratic shape functions and are particularly suitable for solid-state deformation. ${ }^{33}$ SOLID187 describes 10node tetrahedral elements, whereas SOLID186 describes 20-node tetrahedral elements and was primarily used at the level of the drill holes using the finer meshing.

In order to keep the computing time to a minimum, the striking positions have finer meshing than the other areas of the prosthesis. The prosthesis was meshed with a total of 190,000 nodes and 85,000 elements. Figure 4 shows the subareas with their different element sizes.

During the model evolution process two simpler models were attempted, but though increasing in complexity both were still two-dimensional testing models.

The FE model III presented here was then chosen as it represents the most complex.

\section{FE model III}

The most complex model in this study analyzes forces in three dimensions. Based on the results of Duda et al. ${ }^{34}$ Speirs et al. ${ }^{35}$ developed a simplified muscle system model that describes the load of the femur during walking. This FE model (about $25 \%$ of the gait cycle) is specifically designed for the left leg with the maximum muscle force. ${ }^{31}$ The system includes the main muscle groups - abductors, tensor fascia latae, and vastus lateralis - that interact with the femur. The entire bone is used for fixation of the model. In this case, the node P0 receives a fixed bearing and the further four points on the condyle were provided with a floating bearing. Thus, displacements in the $\mathrm{x}$ - or $\mathrm{y}$-direction are excluded. The motions are limited to the $\mathrm{z}$-axis, like the real model, in which the head is stabilized by the acetabulum. The position and fixation of the LISS plate as well as the fracture position are the same as in the earlier FE models creating a Vancouver B 1 fracture below the tip of the prosthesis and screw fixation of the plate.

\section{FE reference model}

A reference model is created using the final model III, which describes the load on an implanted prosthetic socket without drill holes in the prosthesis. This allows

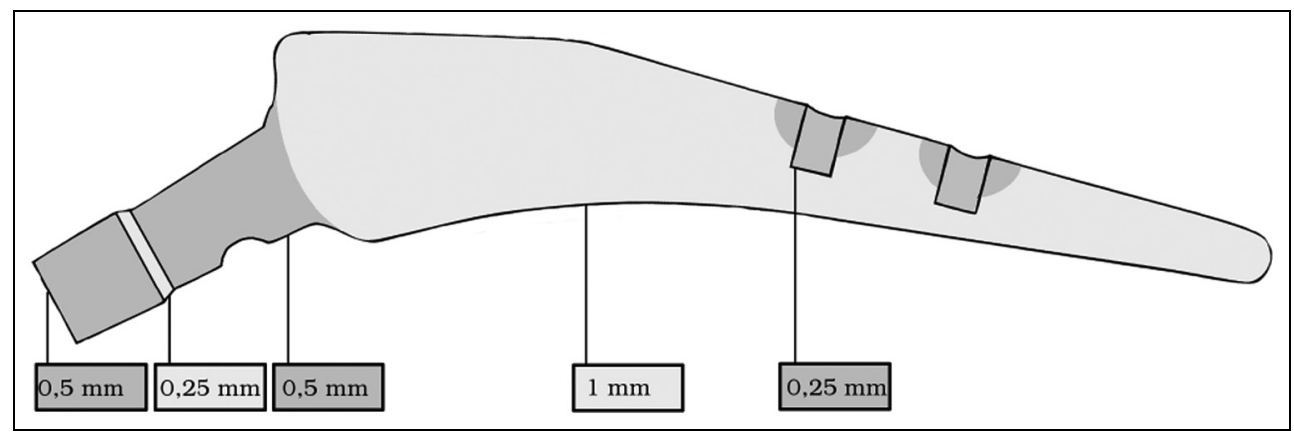

Figure 4. Various subareas of the FE model with corresponding crosslinking sizes. 


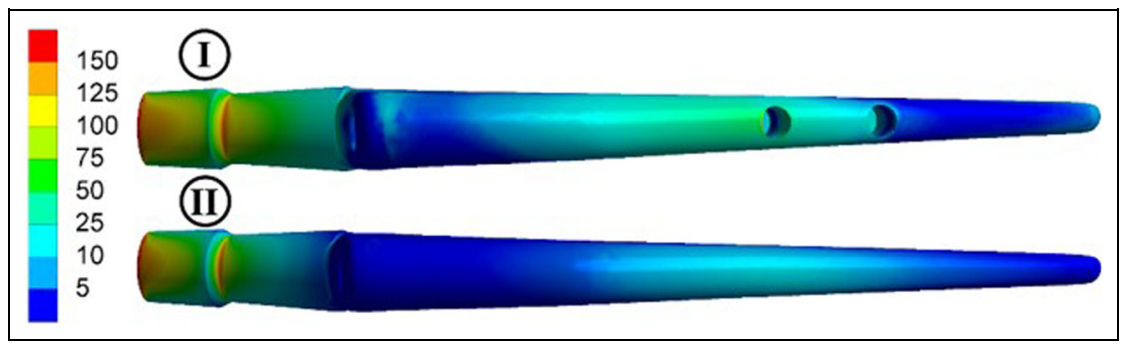

Figure 5. (I) Drilled prosthesis and (II) non-drilled prosthesis as the reference.

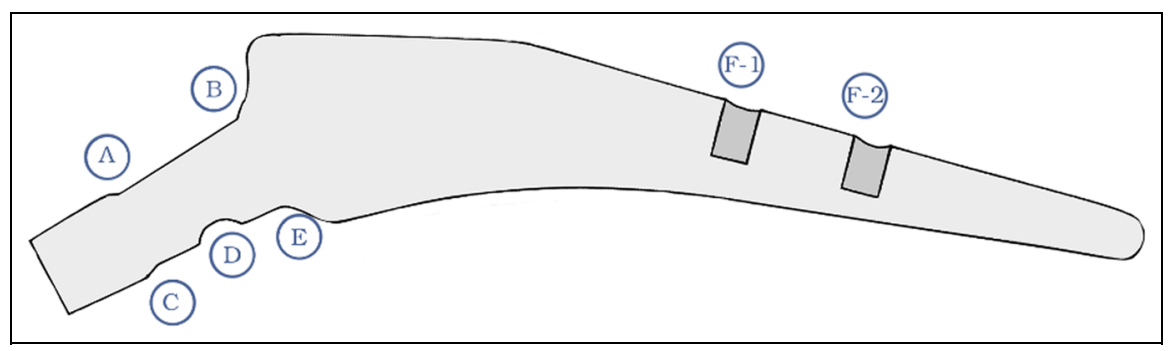

Figure 6. Overview-positions of critical stresses.

a direct comparison between a drilled and undrilled prosthesis. Consequently, it can be used as a general assessment of this osteosynthesis fixation method.

In the construction of the reference model, the LISS plate, the pins, the holes, and the fracture are removed from model III. However, the system of forces remains identical to the existing model. Model III was then modified for the meshing of model components in the area where the holes previously existed. Figure 5 shows the stresses of the drilled (I) and undrilled (II) shaft of the prosthesis.

\section{Variation of the drilling position}

Figure 1 shows the definition of the relevant drilling area.

The curved surface of the greater trochanter led to different incidence angles. The incident angles must be uniform across the models for comparability. The position $\mathrm{a}_{1}$ limits the upper range. At the bottom, the wall thickness limits the range. At the position $\mathrm{a}_{2}$, the wall thickness is $0.5 \mathrm{~mm}$ and is defined as the minimum. Thus, the study area for the holes is $60 \mathrm{~mm}$.

Furthermore, Figure 1 shows two different experimental setups. The distance between the two holes is $40 \mathrm{~mm}$ in experimental setup 2.1 and $20 \mathrm{~mm}$ in testing trial 2.2. The positioning of the pins along the shaft from $\mathrm{a}_{1}$ to $\mathrm{a}_{2}$ ranged in 5 -mm steps.

In the FE simulation, a simplification of the boundary condition is made.

In reality, the LISS plate would have to be repositioned after every step. Due to the large amount of data at the beginning, the influence of LISS plate was examined and only a negligible influence on the simulation results was observed. Therefore, a fixed position was chosen and only the positions of the pins were changed for the simulation.

\section{Results}

For a consistent evaluation of the results, specific positions on the shaft are defined as shown in Figure 6.

Von Mises equivalent stresses are evaluated and the resulting mechanical loads displayed visually based on color. The load is calculated with Von Mises equivalent stresses, equation (1) below: ${ }^{36}$

$$
\sigma_{v}=\sqrt{\frac{1}{2}\left[\left(\sigma_{I}-\sigma_{I I}\right)^{2}+\left(\sigma_{I I}-\sigma_{I I I}\right)^{2}+\left(\sigma_{I I I}-\sigma_{I}\right)^{2}\right]}
$$

The maximum value of the scale is set specifically below the maximum allowable stress. All areas with stress values above the specified maximum are assigned to the color red.

\section{FE model III}

Only a minimal amount of movement occurs between the fracture surfaces by fixing the femoral head. Loading of the femoral head resulted in contactinduced stress at the head area of the prosthesis reaching up to $350 \mathrm{MPa}$. Position A showed the maximum stress of about $205 \mathrm{MPa}$; position $\mathrm{C}$ had the second highest stress threshold of $150 \mathrm{MPa}$. The neck area was loaded up to $100 \mathrm{MPa}$ and the stress level around the drill holes ranged between 30 and $135 \mathrm{MPa}$ depending on the position (Figures 7 and 8).

\section{Variation of drilling position}

Two different concepts (2.1 and 2.2) for variation of the drilling position were evaluated (Figure 8). 


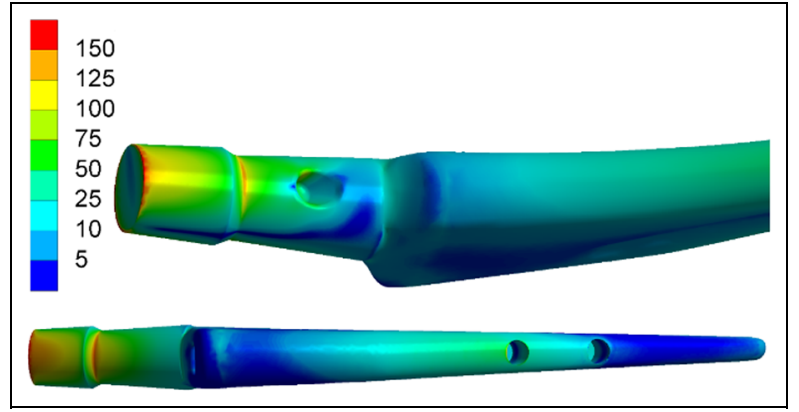

Figure 7. Visual representation of the stresses in model III.

The stress level increased with progressively longer distance from the head to the first drilling position on the neck portion of the prosthesis in both configurations. Similarities in the shape of the curves were observed in both drilling concepts.

A decrease in the load on the prosthesis holes (F-1 and F-2) with increasing distance from the head to the first drilling position was observed shifting to the tip of the prosthesis.

Yield stresses exceeding the ones of the used titanium alloy (924 MPa) were not observed in any drilling position.

A lower load at the neck of the reference model compared to the drilled model was observed. The maximum stress at position $\mathrm{A}$ is $190 \mathrm{MPa}$, which is about $15 \mathrm{MPa}$ lower than the drilled model. The same applies to the positions C (131-150 MPa) and D (82-100 MPa). The stem area of the prosthesis showed a maximum stress of 45-90 MPa.

The stress levels at the prosthesis increased with the number of drilled holes.

\section{Discussion}

The most important finding of this study was that mechanical stresses after drilling the prosthesis remained below the load limit of the material of the hip stem. All deformations of the prosthesis stem remained in the elastic range.

In this study, the load on the implanted hip endoprosthesis was examined after a periprosthetic fracture with using an FE analysis software ANSYS. A model was created, which maps the loaded femur as closely as possible. For this purpose, three SolidWorks models were constructed which differed in structure and boundary conditions. The final model presented a much more complex system than the initial simplified models and is based on a slightly simplified muscle model of the femur.

\section{FE model III}

When comparing the three models, there are several weaknesses in model I and model II. In considering the load of the drilled intraprosthetic hip replacement, model III is far superior. Due to the forces being applied to the femoral head in various directions,
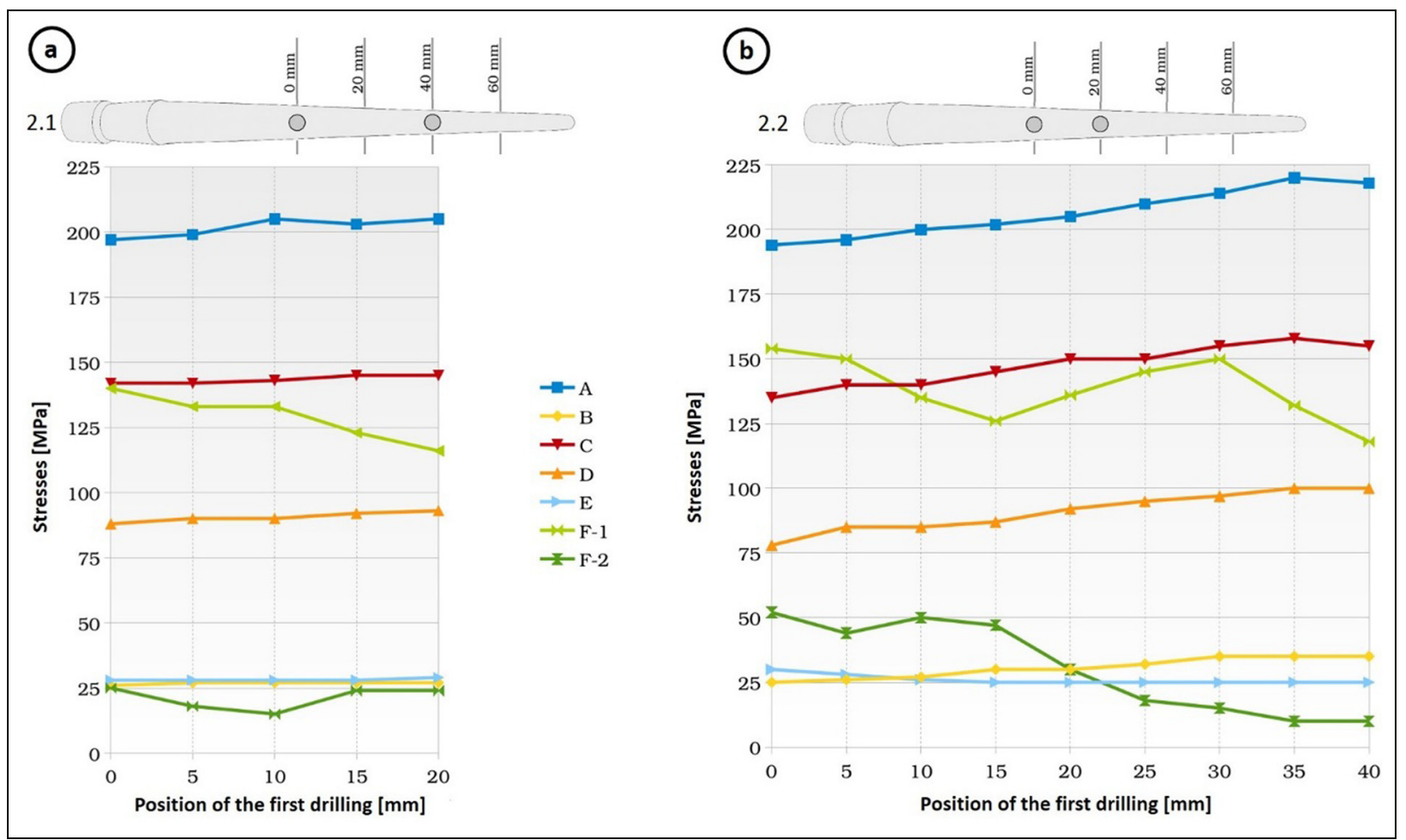

Figure 8. Overview of the prosthesis load as a function of the drilling position.

$\mathrm{FI}$ and $\mathrm{F} 2$ are stresses at the level of the bore holes; $\mathrm{A}$ is the maximum stress position at the proximal prothesial neck; $\mathrm{B}$ is at the level of the prothesial shoulder; $C$ is at the level of the prothesial head; $D$ is the medial border of the prothesial neck; $E$ is at the neck/body junction of the prosthesis. 
model III results in a more accurate understanding of how the implant would react.

The study of the so-called risk bores showed that there are significant changes in the stresses. While the stresses at the site of the risk bore decreased, the load increased in the surrounding stem material. This effect is more pronounced and further the holes were apart, and it was found that increasing the number of holes could counteract this.

The FE reference model therefore without drill holes showed similar stress levels at the head-neck junction compared to our FE model III. At the stem area, the stress levels were lower than that of the drilled model and the trend of the stress levels was harmonious.

But to summarize, no mechanical stresses above the load limit of titanium alloy TiAl6V4 could be detected in any test; all deformations of the prosthesis stem were in the elastic range. The hypothesis was confirmed. These results suggest that using the stable prosthesis for an intraprosthetic screw fixation may be a promising approach to achieve higher primary stability.

This method could be used to study other prosthesis materials, such a cobalt alloy CoCrMo. Furthermore, the fracture mode may also be varied to simulate different loads. As a next step, the drilling machine with the intraprosthetic drilling setup should be integrated into a larger cadaver study to simulate early steps of in vivo use. Also, the impact of eccentric drilling on implant stability has to be investigated in a next step using an FE analysis.

As a major limitation of this study, it can be seen that the results are only carried out statically relying on an FE analysis.

Furthermore, the results achieved with this testing setup are only valid for the specific type of prosthesis tested. Regarding the vast variety of femoral stems, our results might not be directly transferable to all models on the market.

\section{Conclusion}

This FE analysis investigating intraprosthetic screw fixation for periprosthetic femur fractures revealed that mechanical stresses after drilling the prosthesis remained below the load limit of the material of the hip stem (titanium alloy TiAl6V4). All deformations of the prosthesis stem remained in the elastic range, indicating a potential role for this technique in the future treatment of periprosthetic fractures.

\section{Acknowledgements}

Stephan Brand and Michael Bauer have contributed equally to this work.

\section{Declaration of conflicting interests}

The author(s) declared no potential conflicts of interest with respect to the research, authorship, and/or publication of this article.

\section{Funding}

The author(s) received no financial support for the research, authorship, and/or publication of this article.

\section{References}

1. Lindahl H. Epidemiology of periprosthetic femur fracture around a total hip arthroplasty. Injury 2007; 38(6): 651-654.

2. Lewallen DG and Berry DJ. Periprosthetic fracture of the femur after total hip arthroplasty: treatment and results to date. Instr Course Lect 1998; 47: 243-249.

3. Rayan F and Haddad F. Periprosthetic femoral fractures in total hip arthroplasty - a review. Hip Int 2010; 20(4): 418-426.

4. Kavanagh BF. Femoral fractures associated with total hip arthroplasty. Orthop Clin North Am 1992; 23(2): 249 257.

5. Della Rocca GJ, Leung KS and Pape HC. Periprosthetic fractures: epidemiology and future projections. J Orthop Trauma 2011; 25(Suppl. 1): S66-S70.

6. Bhattacharyya T, Chang D, Meigs JB, et al. Mortality after periprosthetic fracture of the femur. $J$ Bone Joint Surg Am 2007; 89(12): 2658-2662.

7. Franklin $\mathbf{J}$ and Malchau H. Risk factors for periprosthetic femoral fracture. Injury 2007; 38(6): 655-660.

8. Ricci WM and Haidukewych GJ. Periprosthetic femoral fractures. Instr Course Lect 2009; 58: 105-115.

9. Klein GR, Parvizi J, Rapuri V, et al. Proximal femoral replacement for the treatment of periprosthetic fractures. J Bone Joint Surg Am 2005; 87(8): 1777-1781.

10. Kolstad K. Revision THR after periprosthetic femoral fractures. An analysis of 23 cases. Acta Orthop Scand 1994; 65(5): 505-508.

11. Ogawa H, Ito Y, Takigami I, et al. Revision total hip arthroplasty for a Vancouver type B3 periprosthetic fracture using an allograft-cemented stem composite by the telescoping technique. $J$ Arthroplasty 2011; 26(4): 665.e25-665.e28.

12. Rayan F, Konan S and Haddad FS. Uncemented revision hip arthroplasty in B2 and B3 periprosthetic femoral fractures-a prospective analysis. Hip Int 2010; 20(1): 3842.

13. Ries MD. Intraoperative modular stem lengthening to treat periprosthetic femur fracture. J Arthroplasty 1996; 11(2): 204-205.

14. Springer BD, Berry DJ and Lewallen DG. Treatment of periprosthetic femoral fractures following total hip arthroplasty with femoral component revision. $J$ Bone Joint Surg Am 2003; 85(11): 2156-2162.

15. Tsiridis E, Narvani AA, Haddad FS, et al. Impaction femoral allografting and cemented revision for periprosthetic femoral fractures. J Bone Joint Surg Br 2004; 86(8): 1124-1132.

16. Frankie L and Xiang Z. Locking compression plate fixation for periprosthetic femoral fracture. Zhongguo Xiu Fu Chong Jian Wai Ke Za Zhi 2002; 16(2): 123-125.

17. Kaab MJ, Stockle U, Schutz M, et al. Stabilisation of periprosthetic fractures with angular stable internal fixation: a report of 13 cases. Arch Orthop Trauma Surg 2006; 126(2): 105-110.

18. Radcliffe SN and Smith DN. The Mennen plate in periprosthetic hip fractures. Injury 1996; 27(1): 27-30. 
19. Ricci WM, Bolhofner BR, Loftus T, et al. Indirect reduction and plate fixation, without grafting, for periprosthetic femoral shaft fractures about a stable intramedullary implant. Surgical technique. J Bone Joint Surg Am 2006; 88(Suppl. 1 Pt 2): 275-282.

20. Ricci WM, Loftus T, Cox C, et al. Locked plates combined with minimally invasive insertion technique for the treatment of periprosthetic supracondylar femur fractures above a total knee arthroplasty. J Orthop Trauma 2006; 20(3): 190-196.

21. Tarnowski JR and Holck K. Osteosynthesis of a periprosthetic fracture of the proximal femur with the distal femur LISS system. Acta Orthop Belg 2008; 74(1): 125-127.

22. Haddad FS, Marston RA and Muirhead-Allwood SK. The Dall-Miles cable and plate system for periprosthetic femoral fractures. Injury 1997; 28(7): 445-447.

23. Tadross TS, Nanu AM, Buchanan MJ, et al. Dall-Miles plating for periprosthetic B1 fractures of the femur. $J$ Arthroplasty 2000; 15(1): 47-51.

24. Venu KM, Koka R, Garikipati R, et al. Dall-Miles cable and plate fixation for the treatment of peri-prosthetic femoral fractures-analysis of results in 13 cases. Injury 2001; 32(5): 395-400.

25. Pafilas D and Kourtzis N. Hybrid external fixation as a new treatment method for periprosthetic femoral fracture. A case report. J Bone Joint Surg Am 2006; 88(1): 188-192.

26. Sakai T, Ohzono K, Nakase T, et al. Treatment of periprosthetic femoral fracture after cementless total hip arthroplasty with Ilizarov external fixation. J Arthroplasty 2007; 22(4): 617-620.

27. Brand S, Klotz J, Hassel T, et al. Intraprosthetic fixation techniques in the treatment of periprosthetic fractures - a biomechanical study. World J Orthop 2012; 3(10): 162-166.

28. Brand S, Klotz J, Hassel T, et al. Intraprosthetic screw fixation increases primary fixation stability in periprosthetic fractures of the femur - a biomechanical study. Med Eng Phys 2014; 36(2): 239-243.

29. Brand S, Klotz J, Hassel T, et al. Different thermal conductivity in drilling of cemented compared with cementless hip prostheses in the treatment of periprosthetic fractures of the proximal femur: an experimental biomechanical analysis. Int Orthop 2013; 37(10): 1885-1889.

30. Brand S, Klotz J, Petri M, et al. Temperature control with internally applied cooling in solid material drilling: an experimental, biomechanical study. Int Orthop 2013; 37(7): 1355-1361.

31. Wang MY, Flanagan SP, Song JE, et al. Relationships among body weight, joint moments generated during functional activities, and hip bone mass in older adults. Clin Biomech 2006; 21(7): 717-725.

32. Claes L, Kirschner P, Perka C, et al. Manual of total hip arthroplasty and revision. Berlin, Heidelberg: Springer, 2012.

33. Barbero EJ. Finite element analysis of composite materials using $A N S Y S^{\circledR}$. Boca Raton, FL: CRC Press, 2014.

34. Duda GN, Heller M, Albinger J, et al. Influence of muscle forces on femoral strain distribution. J Biomech 1998; 31(9): 841-846.

35. Speirs AD, Heller MO, Duda GN, et al. Physiologically based boundary conditions in finite element modelling. $J$ Biomech 2007; 40(10): 2318-2323.

36. Hill R. The mathematical theory of plasticity (Oxford classical texts in physical sciences). New York: Oxford University Press, 1950. 